\title{
Emerging Translational Variance: Vacuum Polarization Energy of the $\phi^{6}$ Kink
}

\author{
H. Weigel \\ Institute for Theoretical Physics, Physics Department, Stellenbosch University, Matieland 7602, South Africa \\ Correspondence should be addressed to H. Weigel; weigel@sun.ac.za
}

Received 19 May 2017; Accepted 8 June 2017; Published 30 July 2017

Academic Editor: Ralf Hofmann

Copyright (C) 2017 H. Weigel. This is an open access article distributed under the Creative Commons Attribution License, which permits unrestricted use, distribution, and reproduction in any medium, provided the original work is properly cited. The publication of this article was funded by SCOAP . $^{3}$

We propose an efficient method to compute the vacuum polarization energy of static field configurations that do not allow decomposition into symmetric and antisymmetric channels in one space dimension. In particular, we compute the vacuum polarization energy of the kink soliton in the $\phi^{6}$ model. We link the dependence of this energy on the position of the center of the soliton to the different masses of the quantum fluctuations at negative and positive spatial infinity.

\section{Motivation}

It is of general interest to compute quantum corrections to classical field configurations like soliton solutions that are frequently interpreted as particles. On top of the wish list, we find the energies that predict particle masses. The quantum correction to the energy can be quite significant because the classical field acts as a background that strongly polarizes the spectrum of the quantum fluctuations about it. For that reason, the quantum correction to the classical energy is called vacuum polarization energy (VPE). Here, we will consider the leading (i.e., one-loop) contribution.

Field theories that have classical soliton solutions in various topological sectors deserve particular interest. Solitons from different sectors have unequal winding numbers and the fluctuation spectrum changes significantly from one sector to another. For example, the number of zero modes is linked to the number of (normalizable) zero modes that in turn arise from the symmetries that are spontaneously broken by the soliton. Of course, the pattern of spontaneous symmetry breaking is subject to the topological structure. On the other hand, the winding number is typically identified with the particle number. The prime example is the Skyrme model $[1,2]$ wherein the winding number determines the baryon number [3, 4]. Many properties of baryons have been studied in this soliton model and its generalization in the past [5].
More recently, configurations with very large winding numbers have been investigated [6] and these solutions were identified with nuclei. To obtain a sensible understanding of the predicted nuclear binding energies, it is, of course, important to consider the VPE, in particular when it is expected to strongly depend on the particle number. So far, this has not been attempted for the simple reason that the model is not renormalizable. A rough estimate [7] (see [8] for a general discussion of the quantum corrections of the Skyrmion and further references on the topic) in the context of the $\mathrm{H}$ dibaryon $[9,10]$ suggests that the VPE strongly reduces the binding energy of multibaryon states.

As already mentioned, one issue for the calculation of the VPE is renormalization. Another important one is, as will be discussed below, that the VPE is (numerically) extracted from the scattering data for the quantum fluctuations about the classical configuration [11]. Though this so-called spectral method allows for direct implementation of standard renormalization conditions, it has limitations as it requires sufficient symmetry for partial wave decomposition. This may not be possible for configurations with an intricate topological structure associated with large winding numbers.

The $\phi^{6}$ model in $D=1+1$ dimensions has soliton solutions with different topological structures $[12,13]$ and the fluctuations do not decouple into parity channels. The approach employed here is also based on scattering data but advances 
the spectral method such that no parity decomposition is required. We will also see that it is significantly more effective than previous computations [14-16] for the VPE of solitons in $D=1+1$ dimensions that are based on heat kernel expansions combined with $\zeta$-function regularization techniques [17-19].

Although the $\phi^{6}$ model is not fully renormalizable, at one-loop order, the ultraviolet divergences can be removed unambiguously. However, another very interesting phenomenon emerges. The distinct topological structures induce nonequivalent vacua that manifest themselves via different dispersion relations for the quantum fluctuations at positive and negative spatial infinity. At some intermediate position, the soliton mediates between these vacua. Since this position cannot be uniquely determined, the resulting VPE exhibits a translational variance. This is surprising since, after all, the model is defined through a local and translational invariant Lagrangian. In this paper, we will describe the emergence of this variance and link it to the different level densities that arise from the dispersion relations. To open these results for discussion (the present paper reflects the author's invited presentation at the 5th Winter Workshop on Non-Perturbative Quantum Field Theory based on the methods derived in [20] making some overlap unavoidable), it is necessary to review in detail the methods developed in [20] to compute the VPE for backgrounds in one space dimension that are not (manifestly) invariant under spatial reflection.

Following this introductory motivation, we will describe the $\phi^{6}$ model and its kink solutions. In Section 3, we will review the spectral method that ultimately leads to a variant of the Krein-Friedel-Lloyd formula [21] for the VPE. The novel approach to obtain the relevant scattering data will be discussed in Section 4 and combined with the one-loop renormalization in Section 5. A comparison with known (exact) results will be given in Section 6 while Section 7 contains the predicted VPE for the solitons of the $\phi^{6}$ model. Translational variance of the VPE that emerges from the existence of nonequivalent vacua will be analyzed in Section 8 . We conclude with a short summary in Section 9.

\section{Kinks in $\phi^{6}$ Models}

In $D=1+1$ dimensions, thedynamics for the quantum field $\phi$ are governed solely by a field potential $U(\phi)$ that is added to the kinetic term

$$
\mathscr{L}=\frac{1}{2} \partial_{\mu} \phi \partial^{\mu} \phi-U(\phi) .
$$

For the $\phi^{6}$ model, we scale all coordinates, fields, and coupling constants such that the potential contains only a single dimensionless parameter $a$ :

$$
U(\phi)=\frac{1}{2}\left(\phi^{2}+a^{2}\right)\left(\phi^{2}-1\right)^{2} .
$$

From Figure 1, we observe that there are three general cases. For $a^{2}>1 / 2$, two degenerate minima at $\phi= \pm 1$ exist. For $0<a^{2} \leq 1 / 2$, an additional local minimum emerges at $\phi=$ 0 . Finally, for $a=0$, the three minima at $\phi=0$ and $\phi=$ \pm 1 are degenerate. Soliton solutions connect different vacua between negative and positive spatial infinity. For $a \neq 0$, the vacua are at $\phi= \pm 1$ and the corresponding soliton solution is [12]

$$
\phi_{K}(x)=a \frac{X-1}{\sqrt{4 X+a^{2}(1+X)^{2}}} \text { with } X=\mathrm{e}^{2 \sqrt{1+a^{2}} x} .
$$

Its classical energy is

$$
E_{\mathrm{cl}}(a)=\frac{2-a^{2}}{4} \sqrt{1+a^{2}}+\frac{4 a^{2}+a^{4}}{8} \ln \frac{\sqrt{1+a^{2}}+1}{\sqrt{1+a^{2}}-1} .
$$

The case $a=0$ is actually more interesting because two distinct soliton solutions do exist. The first one connects $\phi=0$ at $x \rightarrow-\infty$ to $\phi=1$ at $x \rightarrow \infty$ :

$$
\phi_{K_{1}}(x)=\frac{1}{\sqrt{1+\mathrm{e}^{-2 x}}}
$$

while the second one interpolates between $\phi=-1$ and $\phi=0$ :

$$
\phi_{K_{2}}(x)=-\frac{1}{\sqrt{1+\mathrm{e}^{2 x}}} .
$$

These soliton configurations are shown in Figure 2. In either case, the classical mass is $E_{\mathrm{cl}}=1 / 4=(1 / 2) \lim _{a \rightarrow 0} E_{\mathrm{cl}}(a)$. This relation for the classical energies reflects the fact that as $a \rightarrow 0$ the solution $\phi_{K}(x)$ disintegrates into two widely separated structures, one corresponding to $\phi_{K_{1}}(x)$ and the other to $\phi_{K_{2}}(x)$.

The computation of the VPE requires the construction of scattering solutions for fluctuations about the soliton. In the harmonic approximation, the fluctuations experience the potential

$$
V(x)=\left.\frac{1}{2} \frac{\partial^{2} U(\phi)}{\partial \phi^{2}}\right|_{\phi=\phi_{\mathrm{sol}}(x)}
$$

generated by the soliton $\left(\phi_{\text {sol }}=\phi_{K}, \phi_{K_{1}}\right.$, or $\left.\phi_{K_{2}}\right)$. These three potentials are shown in Figure 3. For $a \neq 0$, the potential is invariant under $x \leftrightarrow-x$. But the particular case $a \equiv 0$ is not reflection symmetric, though $x \leftrightarrow-x$ swaps the potentials generated by $\phi_{K_{1}}$ and $\phi_{K_{2}}$. The loss of this invariance disables the separation of the fluctuation modes into symmetric and antisymmetric channels, which is the one-dimensional version of a partial wave decomposition. Even more strikingly, the different topological structures in the $a=0$ case cause $\lim _{x \rightarrow-\infty} V(x) \neq \lim _{x \rightarrow \infty} V(x)$, which implies different masses (dispersion relations) for the fluctuations at positive and negative spatial infinity.

\section{Spectral Methods and Vacuum Polarization Energy}

The formula for the VPE (see (13)) can be derived from first principles in quantum field theory by integrating the vacuum matrix element of the energy density operator [22]. It is, however, also illuminative to count the energy levels when summing the changes of the zero point energies. This sum is $\mathcal{O}(\hbar)$ 


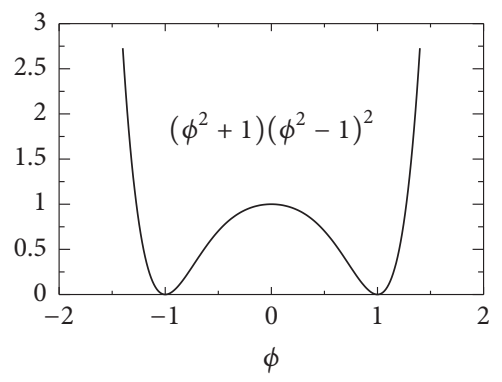

(a)

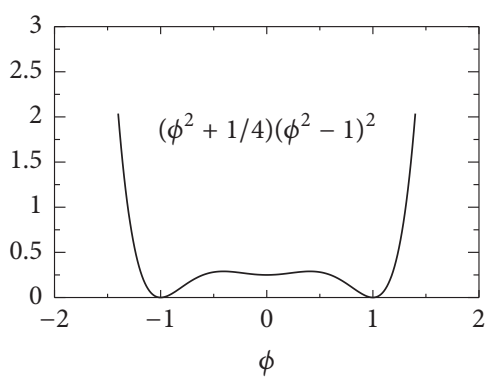

(b)

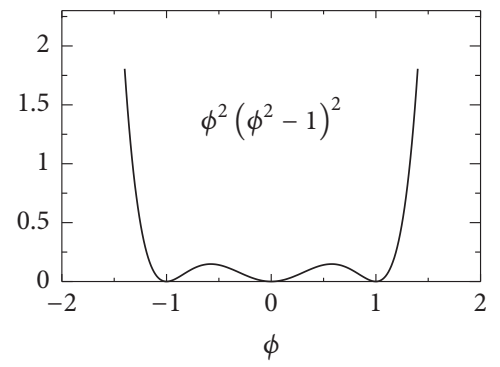

(c)

Figure 1: The field potential (see (2)) in the $\phi^{6}$ model for various values of the real parameter $a=1,1 / 2,0$ from (a) to (c).

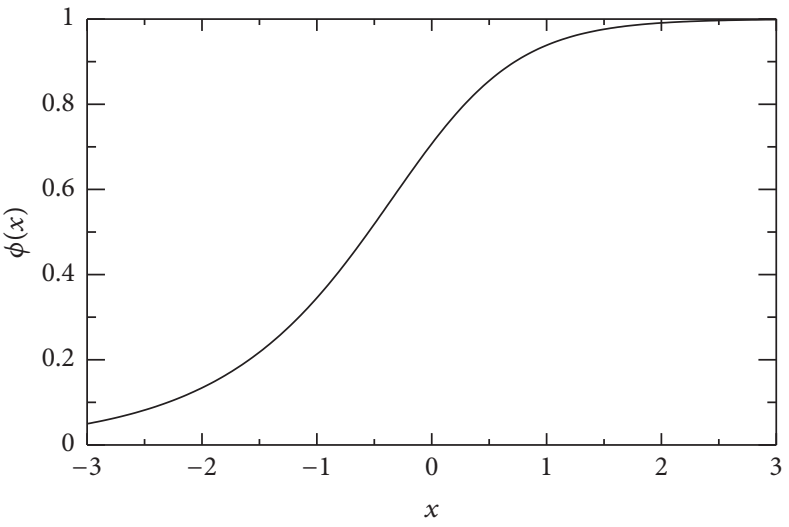

(a)

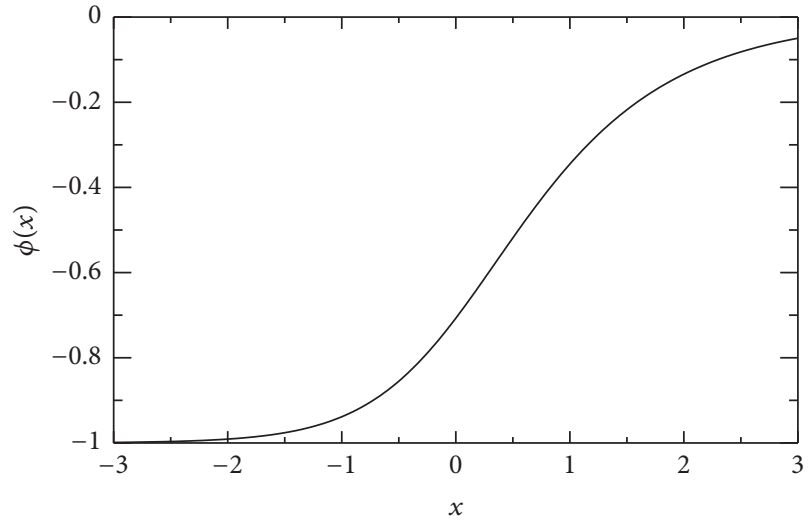

(b)

FIGURE 2: The two soliton solutions for $a=0$ : (a) see (5); (b) see (6).

and thus one-loop order ( $\hbar=1$ for the units used here). We call the single particle energies of fluctuations in the soliton type background $\omega_{n}$ while $\omega_{n}^{(0)}$ are those for the trivial background. Then, the VPE formally reads

$$
\begin{aligned}
E_{\mathrm{vac}} & =\left.\frac{1}{2} \sum_{n}\left(\omega_{n}-\omega_{n}^{(0)}\right)\right|_{\text {ren. }} \\
& =\frac{1}{2} \sum_{j} \epsilon_{j}+\frac{1}{2} \int_{0}^{\infty} d k \omega_{k} \Delta \rho_{\text {ren. }}(k),
\end{aligned}
$$

where the subscript indicates that renormalization is required to obtain a finite and meaningful result. On the right hand side, we have separated the explicit bound state (sum of energies $\epsilon_{j}$ ) and continuum (integral over momentum $k$ ) contributions. The latter involves $\Delta \rho_{\text {ren. }}(k)$ which is the (renormalized) change of the level density induced by the soliton background. Let $L$ be a large distance away from the localized soliton background. For $x \sim L$, the stationary wave function of the quantum fluctuation is a phase shifted plane wave $\psi(x) \sim \sin [k x+\delta(k)]$, where $\delta(k)$ is the phase shift (of a particular partial wave) that is obtained from scattering off the potential (see (7)). The continuum levels are counted from the boundary condition $\psi(L)=0$ and subsequently taking the limit $L \rightarrow \infty$. The number $n(k)$ of levels with momentum less than or equal to $k$ is then extracted from $k L+\delta(k)=n(k) \pi$. The corresponding number in the absence of the soliton is $n^{(0)}(k)=k L / \pi$, trivially. From these, the change of the level density is computed via

$$
\Delta \rho(k)=\lim _{L \rightarrow \infty} \frac{d}{d k}\left[n(k)-n^{(0)}(k)\right]=\frac{1}{\pi} \frac{d \delta(k)}{d k},
$$

which is often referred to as the Krein-Friedel-Lloyd formula [21]. Note that $\Delta \rho(k)$ is a finite quantity; but ultraviolet divergences appear in the momentum integral in (8) and originate from the large $k$ behavior of the phase shift. This behavior is governed by the Born series

$$
\delta(k)=\delta^{(1)}(k)+\delta^{(2)}(k)+\cdots,
$$

where the superscript reflects the power to which the potential (see (7)) contributes. Though this series does not converge (e.g., in three space dimensions, the series yields $\delta(0) \rightarrow 0$ which contradicts Levinson's theorem) for all $k$, it describes the large $k$ behavior well since $\delta^{(N+1)}(k) / \delta^{(N)}(k) \propto 1 / k^{2}$ when $k \rightarrow \infty$. Hence, replacing

$$
\begin{aligned}
& \Delta \rho(k) \longrightarrow[\Delta \rho(k)]_{N} \\
& \quad=\frac{1}{\pi} \frac{d}{d k}\left[\delta(k)-\delta^{(1)}(k)-\delta^{(2)}(k)-\cdots-\delta^{(N)}(k)\right]
\end{aligned}
$$

produces a finite integral in (8) when $N$ is taken sufficiently large. We have to add back the subtractions that come with 


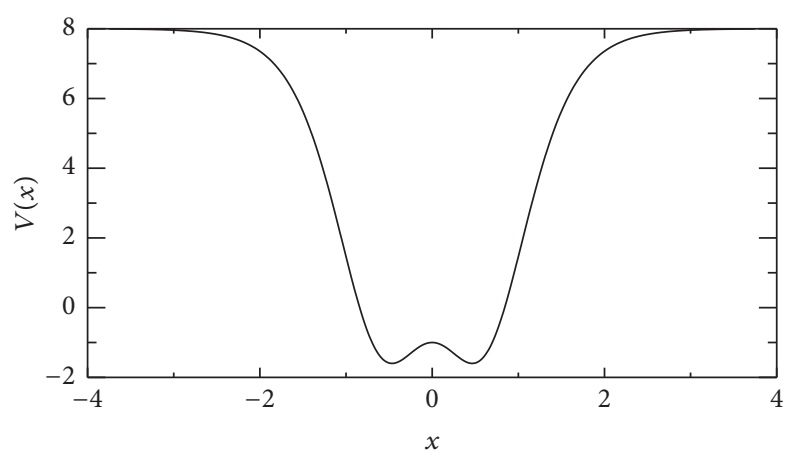

(a)

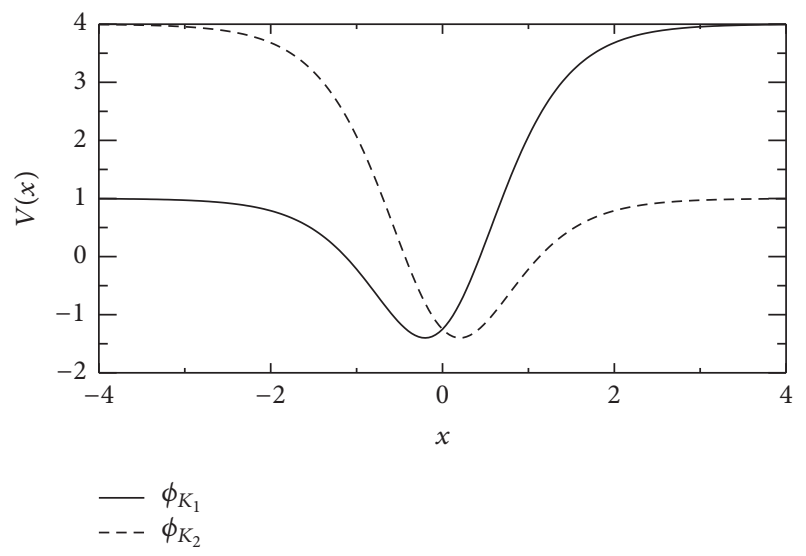

(b)

FIGURE 3: Scattering potentials for the quantum fluctuations in the $\phi^{6}$ model. (a) Typical example for $a \neq 0$; (b) the case $a=0$ with the two potentials generated by $\phi_{K_{1}}$ (full line) and $\phi_{K_{2}}$ (dashed line).

this replacement. Here, the spectral methods take advantage of the fact that each term in the subtraction is uniquely related to a power of the background potential and that Feynman diagrams represent an alternative expansion scheme for the vacuum polarization energy

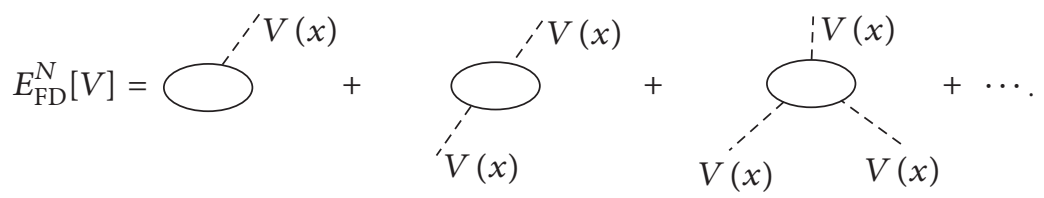

The full lines are the free propagators of the quantum fluctuations and the dashed lines denote insertions of the background potential (see (7)), eventually after Fourier transformation. These Feynman diagrams are regularized with standard techniques, most commonly in dimensional regularization. They can thus be straightforwardly combined with the counterterm contribution, $E_{\mathrm{CT}}[V]$, with coefficients fully determined in the perturbative sector of the theory. This combination remains finite when the regulator is removed.

The generalization to multiple channels is straightforward by finding an eventually momentum dependent diagonalization of the scattering matrix $S(k)$ and summing the so-obtained eigenphase shifts. This replaces $\delta(k) \rightarrow$ $(1 / 2 i) \ln \operatorname{det} S(k)$ (the proper Riemann sheet of the logarithm is identified by constructing a smooth function that vanishes as $k \rightarrow \infty$ ) and analogously for the Born expansion (see (10) and (11)). Since after Born subtraction the integral converges, we integrate by parts to avoid numerical differentiation and to stress that the VPE is measured with respect to the translationally invariant vacuum. We then find the renormalized VPE to be, with the sum over partial waves reinserted,

$$
E_{\mathrm{vac}}[V]=\sum_{\ell} D_{\ell}\left\{\frac{1}{2} \sum_{j}\left(\epsilon_{\ell j}-m\right)\right.
$$

$$
\begin{aligned}
& \left.-\int_{0}^{\infty} \frac{d k}{4 \pi \mathrm{i}} \frac{k}{\sqrt{k^{2}+m^{2}}}[\ln \operatorname{det} S(k)]_{N}\right\}+E_{\mathrm{FD}}^{N}[V] \\
& +E_{\mathrm{CT}}[V] .
\end{aligned}
$$

Here, $D_{\ell}$ is the degree of degeneracy (e.g., $D_{\ell}=2 \ell+1$ in three space dimensions). The subscript $N$ refers to the subtraction of $N$ terms of the Born expansion, as, for example, in (11). We stress that, with $N$ taken sufficiently large, both the expression in curly brackets and the sum $E_{\mathrm{FD}}^{N}[V]+E_{\mathrm{CT}}[V]$ are individually ultraviolet finite and no cutoff parameter is needed [23].

\section{Scattering Data in One Space Dimension}

In this section, we obtain the scattering matrix for general one-dimensional problems and develop an efficient method for its numerical evaluation. This will be at the center of the novel approach to compute the VPE.

We first review the standard approach that is applicable when $V(-x)=V(x)$ (e.g., Figure 3(a)). Then, the partial wave decomposition separates symmetric $\psi_{S}(-x)=\psi_{S}(x)$ and antisymmetric, $\psi_{A}(-x)=-\psi_{A}(x)$ channels. The respective phase shifts can be straightforwardly obtained in a variant of 
the variable phase approach [24] by parameterizing $\psi(x)=$ $\mathrm{e}^{i[k x+\beta(k, x)]}$ and imposing the obvious boundary conditions $\psi_{S}^{\prime}(0)=0$ and $\psi_{A}(0)=0$. (The prime denotes the derivative with respect to $x$.) The wave equation turns into a nonlinear differential equation for the phase function $\beta(k, x)$. When solved subject to $\lim _{x \rightarrow \infty} \beta(k, x)=0$ and $\lim _{x \rightarrow \infty} \beta^{\prime}(k, x)=0$, the scattering matrix is given by [11]

$$
\begin{aligned}
\frac{1}{2 i} \ln \operatorname{det} S(k)= & -2 \operatorname{Re}[\beta(k, 0)] \\
& -\arctan \frac{\operatorname{Im}\left[\beta^{\prime}(k, 0)\right]}{k+\operatorname{Re}\left[\beta^{\prime}(k, 0)\right]} .
\end{aligned}
$$

Linearizing and iterating the differential equation for $\beta(k, x)$ yield the Born series (see (10)). At this point, it is advantageous to use the fact that scattering data can be continued to the upper half complex momentum plane $[25,26]$. That is, when writing $k=i$, the Jost function, whose phase is the scattering phase shift when $k$ is real, is analytic for $\operatorname{Re}[t] \geq 0$. Furthermore, the Jost function has simple zeros at imaginary $k=i \kappa_{j}$ representing the bound states. Formulating the momentum integral from (13) as a contour integral automatically collects the bound state contribution and we obtain a formula as simple as $[11,22]$

$$
\begin{aligned}
E_{\mathrm{vac}}^{(S)}= & \int_{m}^{\infty} \frac{d t}{2 \pi} \frac{t}{\sqrt{t^{2}-m^{2}}} \\
& \times\left[\ln \left\{g(t, 0)\left(g(t, 0)-\frac{1}{t} g^{\prime}(t, 0)\right)\right\}\right]_{N} \\
& +E_{\mathrm{FD}}^{N}[V]+E_{\mathrm{CT}}[V]
\end{aligned}
$$

for the VPE. Here, $g(t, x)$ is the nontrivial factor of the Jost solution whose $x \rightarrow 0$ properties determine the Jost function. The factor function solves the differential equation

$$
g^{\prime \prime}(t, x)=2 \operatorname{tg}^{\prime}(t, x)+V(x) g(t, x),
$$

with the boundary conditions $g(t, \infty)=1$ and $g^{\prime}(t, \infty)=0$; iterating $g(t, x)=1+g^{(1)}(t, x)+g^{(2)}(t, x)+\cdots$ produces the Born series.

In general, however, the potential $V(x)$ is not reflection invariant and no partial wave decomposition is applicable. Even more, there may exist different masses for the quantum fluctuations

$$
\begin{aligned}
& m_{L}^{2}=\lim _{x \rightarrow-\infty} V(x), \\
& m_{R}^{2}=\lim _{x \rightarrow \infty} V(x)
\end{aligned}
$$

as it is the case for the $\phi^{6}$ model with $a=0$ (cf. Figure 3(b)). We adopt the convention that $m_{L} \leq m_{R}$; otherwise, we simply swap $x \rightarrow-x$. Three different cases must be considered. First, above threshold, both momenta $k$ and $q=\sqrt{k^{2}+m_{L}^{2}-m_{R}^{2}}$ are real. To formulate the variable phase approach, we introduce the matching point $x_{m}$ and parameterize

$$
\begin{aligned}
& \psi(x)=A(x) \mathrm{e}^{i k x} \\
& A^{\prime \prime}(x)=-2 i k A^{\prime}(x)+V_{p}(x) A(x) \\
& x \leq x_{m} \\
& \psi(x)=B(x) \mathrm{e}^{i q x} \\
& B^{\prime \prime}(x)=-2 i q B^{\prime}(x)+V_{p}(x) B(x) \\
& x \geq x_{m} .
\end{aligned}
$$

Observe that the pseudopotential

$$
V_{p}(x)=V(x)-m_{L}^{2}+\left(m_{L}^{2}-m_{R}^{2}\right) \Theta\left(x-x_{m}\right)
$$

vanishes at positive and negative spatial infinity. The differential equations (18) are solved for the boundary conditions $A(-\infty)=B(\infty)=1$ and $A^{\prime}(-\infty)=B^{\prime}(\infty)=0$. There are two linearly independent solutions $\psi_{1}$ and $\psi_{2}$ that define the scattering matrix $S=\left(s_{i k}\right)$ via the asymptotic behaviors

$$
\begin{aligned}
& \psi_{1}(x) \sim \begin{cases}\mathrm{e}^{i k x}+s_{12}(k) \mathrm{e}^{-i k x} & \text { as } x \longrightarrow-\infty \\
s_{11}(k) \mathrm{e}^{i q x} & \text { as } x \longrightarrow \infty,\end{cases} \\
& \psi_{2}(x) \sim \begin{cases}s_{22}(k) \mathrm{e}^{-i k x} & \text { as } x \longrightarrow-\infty \\
\mathrm{e}^{-i q x}+s_{21}(k) \mathrm{e}^{i q x} & \text { as } x \longrightarrow \infty .\end{cases}
\end{aligned}
$$

By equating the solutions and their derivatives at $x_{m}$, the scattering matrix is obtained from the factor functions as

$$
\begin{aligned}
& S(k)=\left(\begin{array}{cc}
\mathrm{e}^{-i q x_{m}} & 0 \\
0 & \mathrm{e}^{i k x_{m}}
\end{array}\right)\left(\begin{array}{cc}
B & -A^{*} \\
i q B+B^{\prime} & i k A^{*}-A^{\prime *}
\end{array}\right)^{-1} \\
& \times\left(\begin{array}{cc}
A & -B^{*} \\
i k A+A^{\prime} & i q B^{*}-B^{\prime *}
\end{array}\right)\left(\begin{array}{cc}
\mathrm{e}^{i k x_{m}} & 0 \\
0 & \mathrm{e}^{-i q x_{m}}
\end{array}\right) \\
& \text { for } k \geq \sqrt{m_{R}^{2}-m_{L}^{2}},
\end{aligned}
$$

where $A=A\left(x_{m}\right)$, and so forth. The second case refers to $k \leq \sqrt{m_{R}^{2}-m_{L}^{2}}$ still being real but $q=\mathrm{i} \kappa$ becoming imaginary with $\kappa=\sqrt{m_{R}^{2}-m_{L}^{2}-k^{2}}$. The parameterization of the wave function for $x>x_{m}$ changes to $\psi(x)=B(x) \mathrm{e}^{-\kappa x}$ yielding the differential equation $B^{\prime \prime}(x)=\kappa B^{\prime}(x)+V_{p}(x) B(x)$. The scattering matrix then is a single unitary number

$$
\begin{aligned}
& S(k)=-\frac{A\left(B^{\prime} / B-\kappa-i k\right)-A^{\prime}}{A^{*}\left(B^{\prime} / B-\kappa+i k\right)-A^{\prime *}} \mathrm{e}^{2 i k x_{m}} \\
& \quad \text { for } 0 \leq k \leq \sqrt{m_{R}^{2}-m_{L}^{2}} .
\end{aligned}
$$


It is worth noting that $V_{p} \equiv 0$ corresponds to the step function potential. In that case, the above formalism obviously yields $A \equiv B \equiv 1$ and reproduces the textbook result

$$
\begin{aligned}
& \delta_{\text {step }}(k) \\
& = \begin{cases}(k-q) x_{m}, & \text { for } k \geq \sqrt{m_{R}^{2}-m_{L}^{2}} \\
k x_{m}-\arctan \left(\frac{\sqrt{m_{R}^{2}-m_{L}^{2}-k^{2}}}{k}\right), & \text { for } k \leq \sqrt{m_{R}^{2}-m_{L}^{2}} .\end{cases}
\end{aligned}
$$

In the third regime also $k$ becomes imaginary and we need to identify the bound states energies $\epsilon \leq m_{L}$ that enter (13). We define real variables $\lambda=\sqrt{m_{L}^{2}-\epsilon^{2}}$ and $\kappa(\lambda)=$ $\sqrt{m_{R}^{2}-m_{L}^{2}+\lambda^{2}}$ and solve the wave equation subject to the initial conditions

$$
\begin{aligned}
& \psi_{L}\left(x_{\min }\right)=1, \\
& \psi_{L}^{\prime}\left(x_{\min }\right)=\lambda, \\
& \psi_{R}\left(x_{\max }\right)=1, \\
& \psi_{R}^{\prime}\left(x_{\max }\right)=-\kappa(\lambda),
\end{aligned}
$$

where $x_{\min }$ and $x_{\max }$ represent negative and positive spatial infinity, respectively. Continuity of the wave function requires the Wronskian determinant

$$
\psi_{L}\left(x_{m}\right) \psi_{R}^{\prime}\left(x_{m}\right)-\psi_{R}\left(x_{m}\right) \psi_{L}^{\prime}\left(x_{m}\right) \stackrel{!}{=} 0
$$

to vanish. This occurs only for discrete values $\lambda_{j}$ that in turn determine the bound state energies $\epsilon_{j}=\sqrt{m_{L}^{2}-\lambda_{j}^{2}}$ (the bosonic dispersion relation does not exclude imaginary energies that would hamper the definition of the quantum theory; this case does not occur here).

\section{One-Loop Renormalization in One Space Dimension}

To complete the computation of the VPE, we need to substantiate the renormalization procedure. We commence by identifying the ultraviolet singularities. This is simple in $D=$ $1+1$ dimensions at one-loop order as only the first diagram on the right hand side of (12) is divergent. Furthermore, this diagram is local in the sense that $E_{\mathrm{FD}}^{(1)} \propto(1 / \epsilon) \int d x[V(x)-$ $m_{L}^{2}$ ], where $\epsilon$ is the regulator (e.g., from dimensional regularization). Hence, a counterterm can be constructed that removes not only the singularity but also the diagram in total. This is the so-called no tadpole condition and implies

$$
E_{\mathrm{FD}}^{(1)}+E_{\mathrm{CT}}^{(1)}=0 .
$$

In the next step, we must identify the corresponding Born term in (10). To this end, it is important to note that the counterterm is a functional of the full field $\phi(x)$ that induces the background potential (see (7)). Hence, we must find the Born approximation for $V(x)-m_{L}^{2}$ rather than the one for the pseudopotential $V_{P}(x)$ (see (19)). The standard formulation of the Born approximation as an integral over the potential is, unfortunately, not applicable to $V(x)-m_{L}^{2}$ since it does not vanish at positive spatial infinity. However, we note that $V(x)-m_{L}^{2}=V_{P}(x)+\left(m_{L}^{2}-m_{R}^{2}\right) \Theta\left(x-x_{m}\right)=V_{p}(x)+V_{\text {step }}(x)$ and that, by definition, the first-order correction is linear in the background and thus additive. We may therefore write

$$
\begin{aligned}
\delta^{(1)}(k) & =\delta_{P}^{(1)}(k)+\delta_{\text {step }}^{(1)}(k) \\
& =\left.\frac{-1}{2 k} \int_{-\infty}^{\infty} d x V_{p}(x)\right|_{x_{m}}+\frac{x_{m}}{2 k}\left(m_{L}^{2}-m_{R}^{2}\right) \\
& =\left.\frac{-1}{2 k} \int_{-\infty}^{\infty} d x V_{p}(x)\right|_{0} .
\end{aligned}
$$

The Born approximation for the step function potential has been obtained from the large $k$ expansion of $\delta_{\text {step }}(k)$ in (23). The subscripts in (27) recall that the definition of the pseudopotential (see (19)) induces an implicit dependence on the (artificial) matching point $x_{m}$. Notably, this dependence disappears from the final result. This is the first step towards establishing the matching point independence of the VPE.

The integrals in $E_{\mathrm{FD}}^{(1)}$ and $E_{\mathrm{CT}}^{(1)}$ require further regularization when $m_{L} \neq m_{R}$. In that case, no further finite renormalization beyond the no tadpole condition is realizable.

\section{Comparison with Known Results}

Before presenting detailed numerical results for VPEs, we note that all simulations were verified to produce $S^{\dagger} S=\mathbf{1}$ after attaching pertinent flux factors to the scattering matrix (see (20)). These flux factors are not relevant for the VPE as they multiply to unity under the determinant in (13). In addition, the numerically obtained phase shifts (i.e., $(1 / 2 i) \ln \operatorname{det} S$ ) have been monitored to not vary with $x_{m}$. Since this is also the case for the bound energies, the VPE is verified to be independent of the unrestricted choice for the matching point.

The VPE calculation based on (13) has been applied to the $\phi^{4}$ kink and sine-Gordon soliton models that are defined via the potentials

$$
\begin{aligned}
U_{K}(\phi) & =\frac{1}{2}\left(\phi^{2}-1\right)^{2}, \\
U_{S G}(\phi) & =4(\cos (\phi)-1),
\end{aligned}
$$

respectively. The soliton solutions $\phi_{K}=\tanh \left(x-x_{0}\right)$ and $\phi_{\mathrm{SG}}(x)=4 \arctan \left(\mathrm{e}^{-2\left(x-x_{0}\right)}\right)$ induce the scattering potentials

$$
\begin{aligned}
V_{K}(x)-m^{2} & =6\left[\tanh ^{2}\left(x-x_{0}\right)-1\right], \\
V_{\mathrm{SG}}(x)-m^{2} & =8\left[\tanh ^{2}\left[2\left(x-x_{0}\right)\right]-1\right] .
\end{aligned}
$$

In both cases, we have identical dispersion relations at positive and negative spatial infinity: $m=m_{L}=m_{R}=2$ for the dimensionless units introduced above. The simulation based on (13) reproduces the established results $E_{\mathrm{vac}}^{(K)}=\sqrt{2} / 4-3 / \pi$ and $E_{\mathrm{vac}}^{(\mathrm{SG})}=-2 / \pi$ [27]. These solitons break translational 
invariance spontaneously and thus produce zero mode bound states in the fluctuation spectrum. In addition, the $\phi^{4}$ kink possesses a bound state with energy $\sqrt{3}$ [27]. All bound states are easily observed using (25). The potentials in (29) are reflection symmetric about the soliton center $x_{0}$ and the method of (15) can be straightforwardly applied [11]. However, this method singles out $x_{0}$ (typically set to $x_{0}=0$ ) to determine the boundary condition in the differential equation and therefore cannot be used to establish translational invariance of the VPE. On the contrary, the boundary conditions for (18) are not at all sensitive to $x_{0}$ and we have applied the present method to compute the VPE for various choices of $x_{0}$, all yielding the same numerical result.

The next step is to compute the VPE for asymmetric background potentials that have $m=m_{L}=m_{R}$. For the lack of a soliton model that produces such a potential, we merely consider a two-parameter set of functions

$$
V_{p}(x) \longrightarrow V_{R, \sigma}(x)=A x \mathrm{e}^{-x^{2} / \sigma^{2}}
$$

for the pseudopotential in (18). Although (15) is not directly applicable, it is possible to relate $V_{R, \sigma}(x)$ to the symmetric potential

$$
\begin{aligned}
V_{R}(x) & =A\left[(x+R) \mathrm{e}^{-(x+R)^{2} / \sigma^{2}}-(x-R) \mathrm{e}^{-(x-R)^{2} / \sigma^{2}}\right] \\
& =V_{R}(-x)
\end{aligned}
$$

and apply (15). In the limit $R \rightarrow \infty$, interference effects between the two structures around $x= \pm R$ disappear, resulting in twice the VPE of (30). The numerical comparison is listed in Table 1. Indeed, the two approaches produce identical results as $R \rightarrow \infty$. The symmetrized version converges only slowly for wide potentials (large $\sigma$ ) causing obstacles for the numerical simulation that do not at all occur in the present approach.

\section{Vacuum Polarization Energies in the $\phi^{6}$ Model}

We first discuss the VPE for the $a \neq 0$ case. A typical background potential is shown in Figure 1(a). Obviously, it is reflection invariant and thus the method based on (15) is applicable. In Table 2, we also compare our results to those from the heat kernel expansion of [15] since, to our knowledge, it is the only approach that has also been applied to the asymmetric $a=0$ case in [14]. Not surprisingly, the two methods based on scattering data agree within numerical precision for all values of $a$. The heat kernel results also agree for moderate and large $a$; but for small values, deviations of the order of $10 \%$ are observed. The heat kernel method relies on truncating the expansion of the exact heat kernel about the heat kernel in the absence of a soliton. Although in [15] the expansion has been carried out to the eleventh(!) order, leaving behind a very cumbersome calculation, this does not seem to provide sufficient accuracy for small $a$.

We are now in the position to discuss the VPE for $a=0$ associated with the soliton $\phi_{K_{1}}(x)$ from (5). The potentials for the fluctuations and the resulting scattering data are shown in
Figure 4. By construction, the pseudopotential jumps at $x_{m}=$ 0 . However, neither the phase shift nor the bound state energy (the zero mode is the sole bound state) depends on $x_{m}$. As expected, the phase shift has a threshold cusp at $\sqrt{m_{R}^{2}-m_{L}^{2}}=$ $\sqrt{3}$ and approaches $\pi / 2$ at zero momentum. This is consistent with Levinson's theorem in one space dimension [28] and the fact that there is only a single bound state. In total, we find significant cancellation between the bound state and continuum contributions

$$
E_{\mathrm{vac}}=-0.5+0.4531=-0.0469 .
$$

The result $-0.1264 \sqrt{2}=-0.1788$ (the factor $\sqrt{2}$ is added to adjust the datum from [14] to the present scale) of [14] was estimated relative to $V_{\alpha}(x)=(3 / 2)[1+\tanh (\alpha x)]$ for $\alpha=1$. Our results for various values of $\alpha$ are listed in Table 3 . These results are consistent with $V_{\alpha}(x)$ turning into a step function for large $\alpha$. For the particular value $\alpha=1$, our relative VPE thus is $\Delta E_{\mathrm{vac}}=-0.0469-0.1660=-0.2129$. In view of the results shown in Table 2, especially for small $a$, these data match within the validity of the approximations applied in the heat kernel calculation.

\section{Translational Variance}

So far, we have computed the VPE for the $\phi^{6}$ model soliton centered at $x_{0}=0$. We have already mentioned that there is translational invariance for the VPE of the kink and sine-Gordon solitons. It is also numerically verified for the asymmetric background (see (30)). In those cases, the two vacua at $x \rightarrow \pm \infty$ are equivalent and $q=k$ in (20). When shifting $x \rightarrow x+x_{0}$, the transmission coefficients $\left(s_{11}\right.$ and $\left.s_{22}\right)$ remain unchanged relative to the amplitude of the incoming wave while the reflection coefficients $\left(s_{12}\right.$ and $\left.s_{21}\right)$ acquire opposite phases. Consequently, $\operatorname{det} S$ is invariant. For unequal momenta, this invariance forfeits and the VPE depends on $x_{0}$. This is reflected by the results in Table 4 in which we present the VPE for $V_{\alpha}(x)=(3 / 2)\left[1+\tanh \left(\alpha\left(x+x_{0}\right)\right)\right]$ and the $\phi^{6}$ model soliton $1 / \sqrt{1+\mathrm{e}^{-2\left(x+x_{0}\right)}}$. Obviously, there is a linear dependence of the VPE on $x_{0}$ with the slope insensitive to specific structure of the potential. This insensitivity is consistent with the above remark on the difference between the two momenta. Increasing $x_{0}$ shifts the vacuum with the bigger mass towards negative infinity, thereby removing states from the spectrum and hence decreasing the VPE.

The effect is immediately linked to varying the width of a symmetric barrier potential with height $m_{R}^{2}-m_{L}^{2}=3$ :

$$
V_{\mathrm{SB}}^{\left(x_{0}\right)}(x)=3 \Theta\left(\frac{x_{0}}{2}-|x|\right) .
$$

For this potential, the Jost solution (see (16)) can be obtained analytically [20] and the VPE has the limit

$$
\lim _{x_{0} \rightarrow \infty} \frac{E_{\mathrm{vac}}\left[V_{\mathrm{SB}}^{\left(x_{0}\right)}\right]}{x_{0}} \approx-0.102,
$$

which again reveals the background independent slope observed above. 
TABLE 1: The $R$ dependent data are half the VPE for the symmetrized potential, (31) computed from (15). The data in the column "present" list the results obtained from (13) for the original potential (see (30)).

\begin{tabular}{lccccccc}
\hline$R$ & 1.0 & 1.5 & 2.0 & 2.5 & 3.0 & 3.5 & Present \\
\hline$A=2.5, \sigma=10$ & -0.0369 & -0.0324 & -0.0298 & -0.0294 & -0.0293 & -0.0292 & -0.0293 \\
\hline$R$ & 4.0 & 5.0 & 6.0 & 7.0 & 8.0 & 9.0 & Present \\
\hline$A=0.2, \sigma=4.0$ & -0.0208 & -0.0188 & -0.0170 & -0.0161 & -0.0158 & -0.0157 & -0.0157 \\
\hline
\end{tabular}

TABLE 2: Different methods to compute the VPE of the $\phi^{6}$ soliton for $a \neq 0$.

\begin{tabular}{|c|c|c|c|c|c|c|c|}
\hline$a$ & 0.001 & 0.01 & 0.05 & 0.1 & 0.2 & 1.0 & 1.5 \\
\hline Heat kernel ([15]) & -1.953 & -1.666 & -1.447 & -1.349 & -1.239 & -1.101 & -1.293 \\
\hline Parity sep. (equation (15)) & -2.145 & -1.840 & -1.595 & -1.461 & -1.298 & -1.100 & -1.295 \\
\hline Present (equation (13)) & -2.146 & -1.841 & -1.596 & -1.462 & -1.297 & -1.102 & -1.297 \\
\hline
\end{tabular}

TABLE 3: VPE for background potential $V_{\alpha}(x)$ defined in the main text. The entry "step" gives the VPE for the step function potential $V(x)=3 \Theta(x)$ using $(23)$ and its Born approximation from (27) for $x_{m}=0$.

\begin{tabular}{ccccccc}
\hline$\alpha$ & 1.0 & 2.0 & 5.0 & 10.0 & 30.0 & Step \\
\hline$E_{\text {vac }}$ & 0.1660 & 0.1478 & 0.1385 & 0.1363 & 0.1355 & 0.1355 \\
\hline
\end{tabular}

TABLE 4: The VPE as a function of the position of the center of the potential for $V_{\alpha}$ and the $\phi^{6}$ model soliton. $\Delta E_{\mathrm{vac}}$ is the difference between the VPEs of the latter and $V_{1}$.

\begin{tabular}{lccccc}
\hline$x_{0}$ & -2 & -1 & 0 & 1 & 2 \\
\hline$\alpha=5$ & 0.341 & 0.240 & 0.139 & 0.037 & -0.064 \\
$\alpha=2$ & 0.351 & 0.250 & 0.148 & 0.046 & -0.057 \\
$\alpha=1$ & 0.369 & 0.267 & 0.166 & 0.064 & -0.038 \\
$\phi^{6}$ & 0.154 & 0.053 & -0.047 & -0.148 & -0.249 \\
$\Delta E_{\text {vac }}$ & -0.215 & -0.214 & -0.213 & -0.212 & -0.211 \\
\hline
\end{tabular}

Having quantitatively determined the translation variance of the VPE, it is tempting to subtract $E_{\mathrm{vac}}\left[V_{\mathrm{SB}}^{\left(x_{0}\right)}\right]$. Unfortunately, this is not unique because $x_{0}$ is not the unambiguous center of the soliton. For example, employing the classical energy density $\epsilon(x)$ to define the position of the soliton $1 / \sqrt{1+\mathrm{e}^{-2(x-\bar{x})}}$, which is formally centered at $\bar{x}$, as an expectation value leads to

$$
x_{s}=\frac{\int d x x \epsilon(x)}{\int d x \epsilon(x)}=\bar{x}+\frac{1}{2} \text {. }
$$

This changes the VPE by approximately 0.050 . This ambiguity also hampers the evaluation of the VPE as half that of a widely separated kink-antikink pair

$$
\phi_{K \bar{K}}(x)=\left[1+\mathrm{e}^{2(x-\bar{x})}\right]^{-1 / 2}+\left[1+\mathrm{e}^{-2(x+\bar{x})}\right]^{-1 / 2}-1
$$

similar to the approach for (31). The corresponding background potential $V_{B}$ is shown in Figure 5. For computing the VPE, the large contribution from the constant but nonzero potential in the regime $|x| \leqslant \bar{x}$ should be eliminated. The above considerations lead to

$$
\begin{aligned}
& \frac{1}{2} \lim _{\bar{x} \rightarrow \infty}\left\{E_{\mathrm{vac}}\left[V_{B}\right]-2 E_{\mathrm{vac}}\left[V_{\mathrm{SB}}^{(2 \bar{x})}\right]\right\}=-0.170, \\
& \frac{1}{2} \lim _{\bar{x} \rightarrow \infty}\left\{E_{\mathrm{vac}}\left[V_{B}\right]-2 E_{\mathrm{vac}}\left[V_{\mathrm{SB}}^{\left(2 x_{s}\right)}\right]\right\}=-0.120 .
\end{aligned}
$$

When the VPE from $V_{\mathrm{SB}}^{(2(\bar{x}+1.2))}$ is subtracted, the main result (see (32)) is matched. Eventually, this can be used to define the center of the soliton.

Now, we also understand why the VPE for $a \neq 0$ diverges as $a \rightarrow 0$ (cf. Table 2). In that limit, kink and antikink structures separate and the "vacuum" in between produces an ever-increasing contribution (in magnitude).

Finally, we discuss the link between the translational variance and the Krein-Friedel-Lloyd formula (see (9)). We have already reported the VPE for the step function potential when $x_{m}=0$. We can also consider $x_{m} \rightarrow \infty$ :

$$
\begin{aligned}
& \frac{E_{\mathrm{vac}}\left[V_{\text {step }}^{\left(x_{m}\right)}\right]}{\left|x_{m}\right|} \longrightarrow-\operatorname{sign}\left(x_{m}\right)\left[\int_{0}^{\sqrt{3}} \frac{d k}{4 \pi} \frac{2 k^{2}-3}{\sqrt{k^{2}+1}}\right. \\
& \left.+\int_{\sqrt{3}}^{\infty} \frac{d k}{4 \pi} \frac{2 k^{2}-2 k \sqrt{k^{2}-3}-3}{\sqrt{k^{2}+1}}\right] \approx 0.101 \operatorname{sign}\left(x_{m}\right),
\end{aligned}
$$

reproducing the linear dependence on the position from above. Formally, that is, without Born subtraction, the integral (see (38)) is dominated by

$$
\begin{aligned}
& \int \frac{d k}{2 \pi} \frac{k}{\sqrt{k^{2}+1}}\left[k-\sqrt{k^{2}-3}\right] \\
& \sim \int \frac{d k}{2 \pi} \sqrt{k^{2}+1} \frac{d}{d k}\left[\sqrt{k^{2}-3}-k\right] \\
& \quad=\int \frac{d k}{2 \pi} \sqrt{k^{2}+1} \frac{d}{d k}[q-k] .
\end{aligned}
$$

Essentially, this is that part of the level density that originates from the different dispersion relations at positive and negative spatial infinity. 


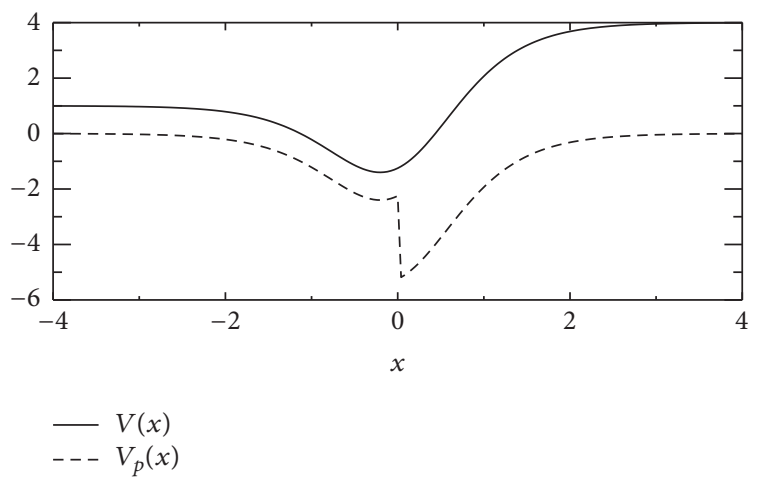

(a)

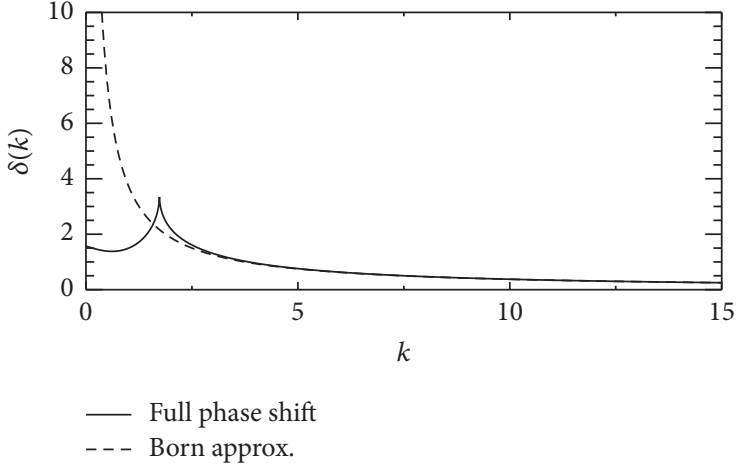

(b)

Figure 4: (a) Potential $(V)$ and pseudopotential $\left(V_{p}\right)$ for fluctuations about a $\phi^{6}$ soliton with $a=0$. The pseudopotential is shown for $x_{m}=0$. (b) Resulting phase shift, that is, $(1 / 2 i) \ln \operatorname{det} S$ (full line), and its Born approximation (dashed line).

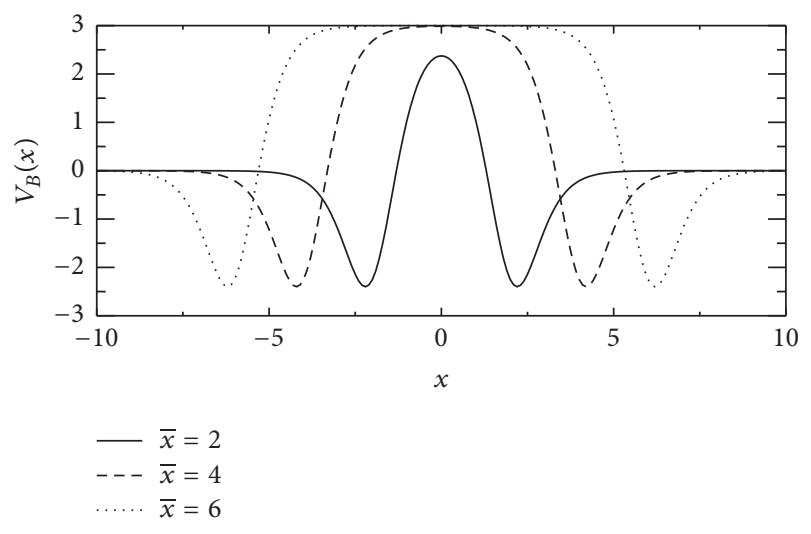

FIGURE 5: Background potential for the kink-antikink pair (see (36)) for different separation instances.

\section{Conclusion}

We have advanced the spectral methods for computing vacuum polarization energies (VPEs) to also apply to static localized background configurations in one space dimension that do not permit a parity decomposition for the quantum fluctuations. The essential progress is the generalization of the variable phase approach to such configurations. Being developed from spectral methods, it adopts their amenities, as, for example, an effective procedure to implement standard renormalization conditions. A glimpse at the bulky formulas for the heat kernel expansion (alternative method to the problem) in [14-16] immediately reveals the simplicity and effectiveness of the present approach. The latter merely requires numerically integrating ordinary differential equations and extracting the scattering matrix thereof (cf. (18) and (21)). Heat kernel methods are typically combined with $\zeta$-function regularization. Then, the connection to standard renormalization conditions is not as transparent as for the spectral methods, though that is problematic only when nonlocal Feynman diagrams require renormalization, that is, in larger than $D=1+1$ dimensions or when fermion loops are involved.

We have verified the novel method by means of wellestablished results, as, for example, the $\phi^{4}$ kink and sineGordon solitons. For these models, the approach directly ascertains translational invariance of the VPE. Yet, the main focus was on the VPE for solitons in $\phi^{6}$ models because its soliton(s) may connect inequivalent vacua leading to background potentials that are not invariant under spatial reflection. This model is not strictly renormalizable. Nevertheless, at one-loop order, a well-defined result can be obtained from the no tadpole renormalization condition although no further finite renormalization is realizable because the different vacua yield additional infinities when integrating the counterterm. The different vacua also lead to different dispersion relations for the quantum fluctuations and thereby induce translational variance for a theory that is formulated by an invariant action. We argue that this variance is universal, as it is not linked to the particular structure of the background and can be related to the change in the level density that is basic to the Krein-Friedel-Lloyd formula (see (9)).

Besides attempting a deeper understanding of the variance by tracing it from the energy momentum tensor, future studies will apply the novel method to solitons of the $\phi^{8}$ model. Its elaborated structure not only induces potentials that are reflection asymmetric but also leads to a set of topological indexes [29] that are related to different particle numbers. Then, the novel method will progress the understanding of quantum corrections to binding energies of compound objects in the soliton picture. Furthermore, the present results can be joined with the interface formalism [30], which augments additional coordinates along which the background is homogeneous, to explore the energy (densities) of domain wall configurations [31].

\section{Disclosure}

This work was presented at the 5th Winter Workshop on Non-Perturbative Quantum Field Theory, Sophia-Antipolis (France), March 2017. 


\section{Conflicts of Interest}

The author declares that there are no conflicts of interest regarding the publication of this paper.

\section{Acknowledgments}

The author is grateful to the organizers for providing this worthwhile workshop. This work is supported in parts by the NRF under Grant 109497.

\section{References}

[1] T. H. R. Skyrme, "A non-linear field theory," Proceedings of the Royal Society A: Mathematical, Physical and Engineering Sciences, vol. 260, pp. 127-138, 1961.

[2] T. H. Skyrme, "The origins of Skyrmions," International Journal of Modern Physics A. Particles and Fields. Gravitation. Cosmology, vol. 3, no. 12, pp. 2745-2751, 1988, (Article reconstructed by I. Aitchison).

[3] E. Witten, "Baryons in the $1 / \mathrm{N}$ expansion," Nuclear Physics B, vol. 160, no. 1, pp. 57-115, 1979.

[4] G. S. Adkins, C. R. Nappi, and E. Witten, "Static properties of nucleons in the Skyrme model," Nuclear Physics, Section B, vol. 228, no. 3, pp. 552-566, 1983.

[5] H. Weigel, vol. 743 of Lecture Notes in Physics, 2008.

[6] D. T. J. Feist, P. H. C. Lau, and N. S. Manton, "Skyrmions up to baryon number 108," Physical Review D, vol. 87, Article ID 085034, 2013.

[7] F. G. Scholtz, B. Schwesinger, and H. B. Geyer, "The Casimir energy of strongly bound $\mathrm{B}=2$ configurations in the Skyrme model," Nuclear Physics, Section A, vol. 561, no. 4, pp. 542-556, 1993.

[8] F. Meier and H. Walliser, "Quantum corrections to baryon properties in chiral soliton models," Physics Reports, vol. 289, no. 6, pp. 383-450, 1997.

[9] R. L. Jaffe, "Perhaps a stable dihyperon," Physical Review Letters, vol. 38, p. 195, 1977, (Erratum: Physical Review Letters vol. 38, p. 617, 1977).

[10] A. P. Balachandran, A. Barducci, F. Lizzi, V. G. J. Rodgers, and A. Stern, "Doubly strange dibaryon in the chiral model," Physical Review Letters, vol. 52, no. 11, pp. 887-890, 1984.

[11] N. Graham, M. Quandt, and H. Weigel, Spectral Methods in Quantum Field Theory, vol. 777 of Lecture Notes in Physics, Springer, Berlin, Germany, 2009.

[12] M. A. Lohe, "Soliton structures in P2," Physical Review D, vol. 20, no. 12, pp. 3120-3130, 1979.

[13] M. A. Lohe and D. M. O’Brien, "Soliton mass corrections and explicit models in two dimensions," Physical Review. D. Particles and Fields. Third Series, vol. 23, no. 8, pp. 1771-1780, 1981.

[14] A. Alonso Izquierdo, W. Garcia Fuertes, M. A. Gonzalez Leon, and J. Mateos Guilarte, "Generalized zeta functions and oneloop corrections to quantum kink masses," Nuclear Physics. B, vol. 635 , no. 3 , pp. 525-557, 2002.

[15] A. Alonso Izquierdo and J. M. Guilarte, "One-loop kink mass shifts: a computational approach," Nuclear Physics B, vol. 852, no. 3, pp. 696-735, 2011.

[16] A. Alonso-Izquierdo and J. Mateos Guilarte, "On a family of (1+1)-dimensional scalar field theory models: kinks, stability, one-loop mass shifts," Annals of Physics, vol. 327, no. 9, pp. 22512274, 2012.
[17] E. Elizalde, Ten Physical Applications of Spectral Zeta Functions, vol. 35 of Lecture Notes in Physics, Springer, Berlin, Germany, 1995.

[18] E. Elizalde, S. D. Odintsov, A. Romeo, A. A. Bytsenko, and S. Zerbini, Zeta Regularization Techniques with Applications, World Scientific Publishing, River Edge, NJ, USA, 1994.

[19] K. Kirsten, "Spectral functions in mathematics and physics," AIP Conference Proceedings, vol. 484, no. 1, p. 106, 1999.

[20] H. Weigel, "Vacuum polarization energy for general backgrounds in one space dimension," Physics Letters B, vol. 766, pp. 65-70, 2017.

[21] J. S. Faulkner, "Scattering theory and cluster calculations," Journal of Physics C: Solid State Physics, vol. 10, no. 23, pp. 46614670, 1977.

[22] N. Graham, R. L. Jaffe, V. Khemani, M. Quandt, M. Scandurra, and H. Weigel, "Calculating vacuum energies in renormalizable quantum field theories: a new approach to the Casimir problem," Nuclear Physics B, vol. 645, no. 3, pp. 49-84, 2002.

[23] E. Farhi, N. Graham, P. Haagensen, and R. L. Jaffe, "Finite quantum fluctuations about static field configurations," Physics Letters B, vol. 427, no. 3-4, pp. 334-342, 1998.

[24] R. Calegero, Variable Phase Approach to Potential Scattering, Academic Press, New York, NY, USA, 1967.

[25] R. G. Newton, Scattering Theory of Waves and Particles, Springer, New York, NY, USA, 2nd edition, 1982.

[26] K. Chadan and P. C. Sabatier, Inverse Problems in Quantum Scattering Theory, Texts and Monographs in Physics, Springer, New York, NY, USA, 2nd edition, 1989.

[27] R. Rajaraman, Solitons and Instantons, North-Holland, Amsterdam, The Netherlands, 1982.

[28] G. Barton, "Levinson's theorem in one dimension: heuristics," Journal of Physics. A. Mathematical and General, vol. 18, no. 3, pp. 479-494, 1985.

[29] V. A. Gani, V. Lensky, and M. A. Lizunova, "Kink excitation spectra in the (1+1)-dimensional," Journal of High Energy Physics, vol. 2015, article 147, 2015.

[30] N. Graham, R. L. Jaffe, M. Quandt, and H. Weigel, "Quantum energies of interfaces," Physical Review Letters, vol. 87, Article ID 131601, 2001.

[31] A. Parnachev and L. G. Yaffe, "One-loop quantum energy densities of domain wall field configurations," Physical Review $D$, vol. 62, Article ID 105034, 2000. 

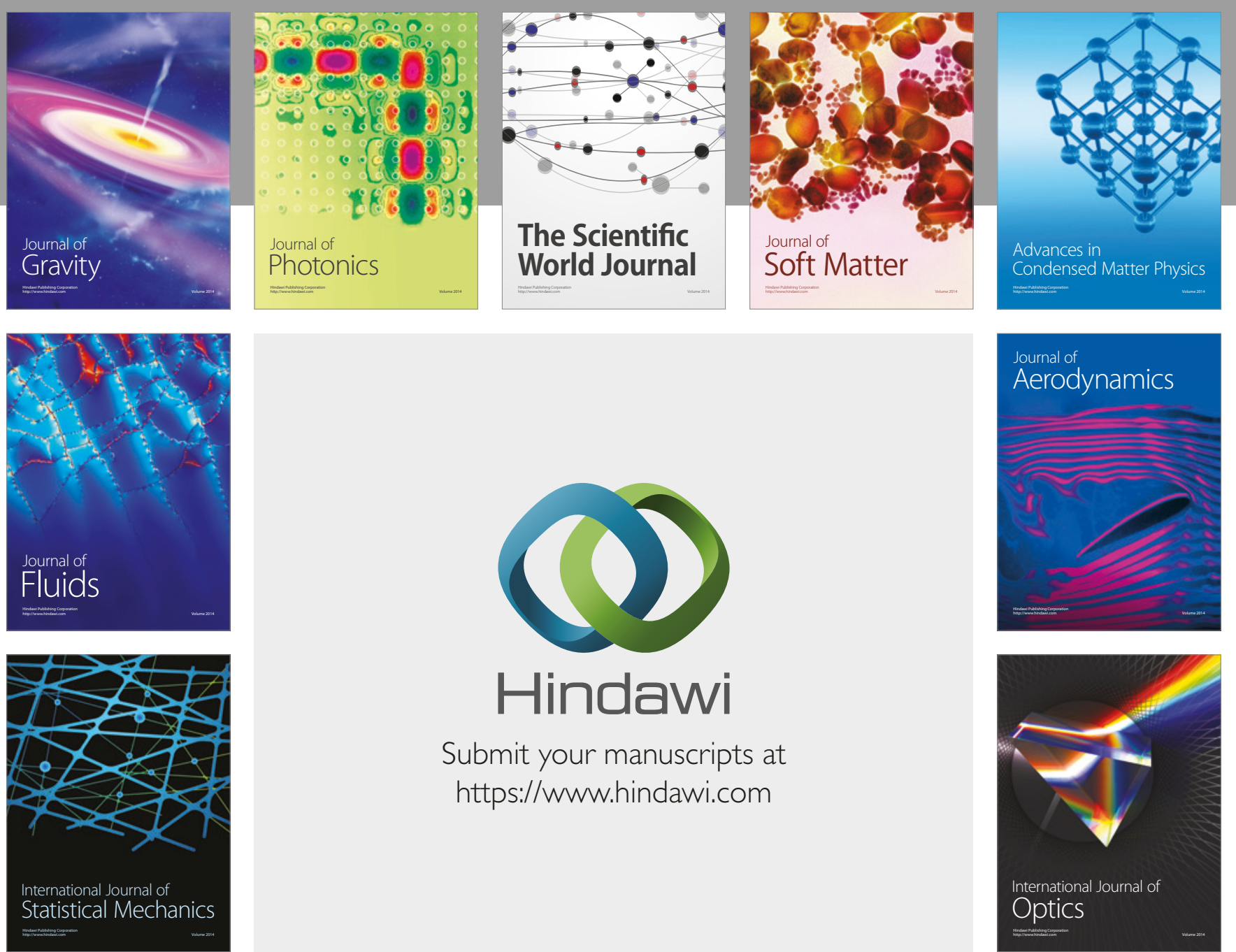

Submit your manuscripts at

https://www.hindawi.com
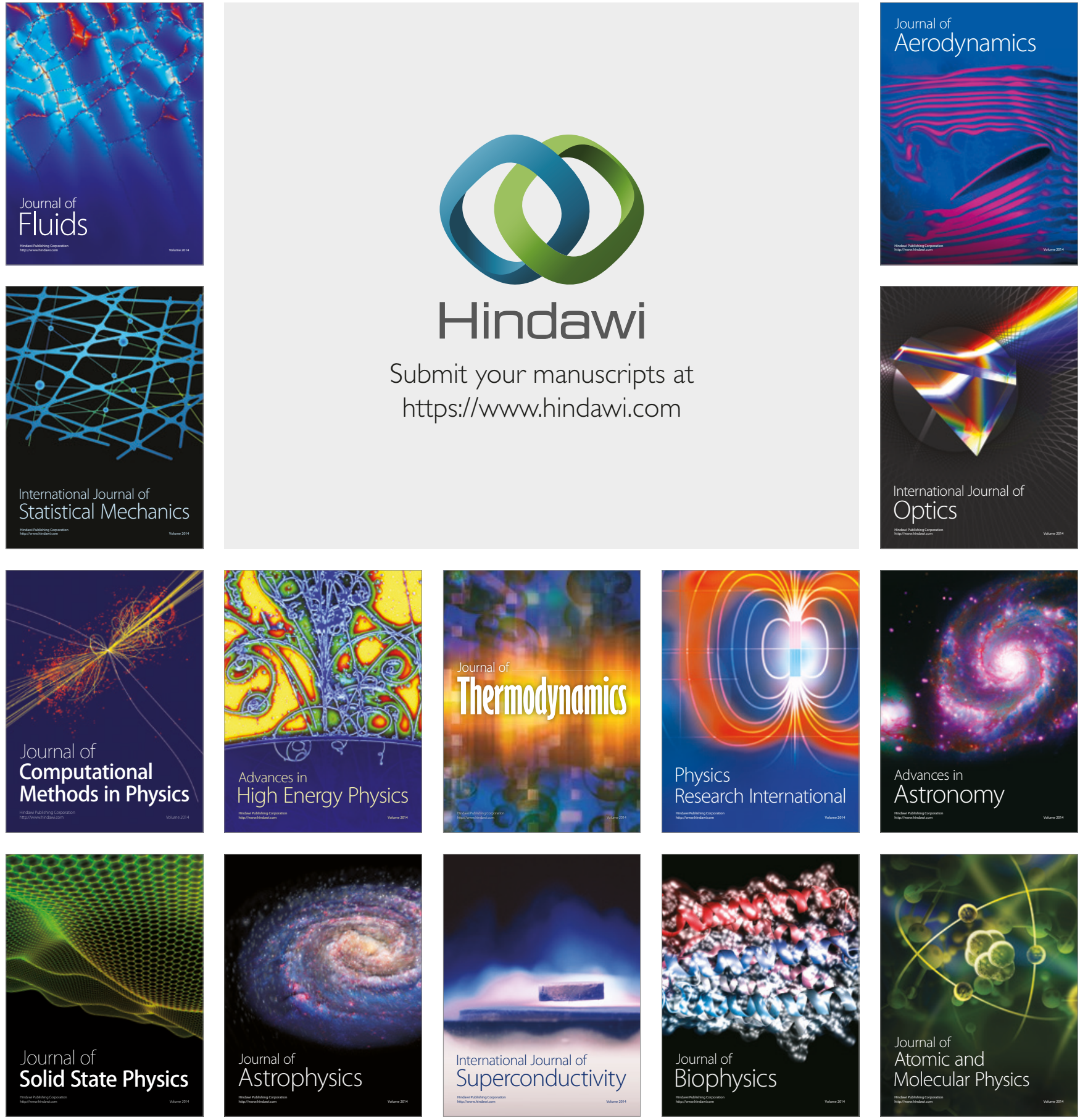